Mitteilungen der Österreichischen Geographischen Gesellschaft, 156. Jg. (Jahresband), Wien 2014, S. 359-363

\title{
ÖSTERREICH-BEZÜGE INTERNATIONALER TOPONOMASTISCHER TAGUNGEN DES JAHRES 2014
}

\author{
Peter JORDAN, Wien* \\ mit 1 Abb. im Text
}

\begin{abstract}
28. Sitzung der Sachverständigengruppe für geographische Namen (United Nations Group of Experts on Geographical Names, UNGEGN), New York, 28. April - 2. Mai 2014
\end{abstract}

Die Vereinten Nationen unterhalten seit 1967 als eine von nur sechs aktiven Expertengruppen eine Sachverständigengruppe für geographische Namen ${ }^{1)}$, die sich in der Regel jedes zweite Jahr trifft, um fachlich zu diskutieren und Resolutionen der Vereinten Nationen im Bereich der geographischen Namen vorzubereiten, die dann bei den fünfjährlich stattfindenden Konferenzen beschlossen werden können. Die Gruppe setzt sich aus Geographen, Kartographen, Geodäten, Linguisten, Historikern und Juristen, aber auch aus Diplomaten zusammen. Die Teilnehmer handeln als Delegierte ihrer jeweiligen Länder und Regierungen und sind diesen gegenüber auch weisungsgebunden. Der Leiter der österreichischen Delegation ist der Vorsitzende der Arbeitsgemeinschaft für Kartographische Ortsnamenkunde (AKO), des österreichischen Expertengremiums für geographische Namen, der von der Österreichischen Akademie der Wissenschaften (ÖAW) nominiert und vom Bundesministerium für Europa, Integration und Äußeres im Namen der österreichischen Bundesregierung delegiert wird. Die Delegation besteht zumeist auch noch aus dem Stellvertretenden Vorsitzenden der AKO, manchmal auch noch aus weiteren Mitgliedern.

Die Sitzung des Jahres 2014 fand im Hauptquartier der Vereinten Nationen am East River in New York statt. 164 Delegierte aus 53 Mitgliedsstaaten der Vereinten Nationen nahmen an ihr teil, außerdem acht Beobachter von privaten Unternehmen wie der Google Corporation und Nicht-Regierungs-Organisationen. Die größten Delegationen stellten die Republik Korea, Indonesien und China mit zwölf bzw. elf und neun Delegierten. Das Gerüst des einwöchigen Sitzungsverlaufs bilden kurze (etwa fünfminütige) Präsentationen von Arbeitspapieren (Working Papers, WP), die schon einen Monat vor der Sitzung auf der UNGEGN-Website zugänglich sind und jeweils im Anschluss an ihre Präsentation im Plenum diskutiert werden. In den Sitzungspausen und an den Abenden finden Business Meetings der thematischen Arbeitsgruppen (Working Groups) und der geographischen oder sprachbezogenen Abteilungen (Divisions) der UNGEGN sowie Präsentationen statt. Bei der 28. Sitzung wurden 80 Arbeitspapiere vorgestellt, zudem gab es sechs ausführlichere Präsentationen.

* Hofrat Prof. h.c. Univ.-Doz. Dr. Peter Jordan, Institut für Stadt- und Regionalforschung, Österreichische Akademie der Wissenschaften, Postgasse 7/4/2,A-1010 Wien; E-Mail: peter.jordan@oeaw.ac.at, http:// www.oeaw.ac.at/isr

1) Website der UNGEGN mit allen Arbeitspapieren und Dokumenten auch der 28. UNGEGN-Sitzung: http://unstats.un.org/unsd/geoinfo/UNGEGN/default.html 
Aus Österreich nahmen der Geograph und Kartograph Peter JoRdan, ÖAW Wien, als Delegationsleiter und der Linguist Gerhard RAMPL, Universität Innsbruck, als Mitglied der Delegation teil. Sie präsentierten insgesamt sieben Arbeitspapiere, zum Teil als Vertreter Österreichs, zum Teil in ihren UNGEGN-Funktionen. In Vertretung Österreichs standen die folgenden Arbeitspapiere auf der Agenda: „Place-names changes in connection with administrative reforms in the Austrian province of Styria“ (Autor: Gernot OBERSTEINER, Steiermärkisches Landesarchiv), „Digitalization of the Franciscan Cadastral Survey (1817-1861) in Austria“ (Autoren: Rupert Kugler \& Franz SchönweILER, Bundesamt für Eich- und Vermessungswesen), „Crowdsourcing and GIS-based methods in a field name survey in Tyrol (Austria)" (Autor: Gerhard RAMPL, Universität Innsbruck), ,Research results from the onomastic project WippDigital - GIS-supported field name research in the Wipptal-area" (Autorin: Daniela FeistmantL, Universität Innsbruck). In seiner neuen, bei dieser Sitzung vom österreichischen Linguisten Hubert BERGMANN übernommenen Funktion des internationalen Koordinators der Toponymic Guidelines for Map and Other Editors for International Use (TG) präsentierte Gerhard RAMPL den „Report of the coordinator". Peter JORDAN übernahm als nun schon seit acht Jahren amtierender Convenor der UNGEGN-Arbeitsgruppe für Exonyme wieder die Präsentation des „Report of the Working Group on Exonyms“ sowie die Vorstellung des Tagungsbandes der letzten ArbeitsgruppenTagung auf Korfu [Kérkyra] mit dem Titel „The Quest for Definitions“.

JORDAN war außerdem an zwei der zahlreichen Nebensitzungen gestaltend beteiligt: am Business Meeting der von ihm geleiteten Arbeitsgruppe für Exonyme sowie an der Präsentation der Joint ICA/IGU Working Group/Commission on Toponymy, während der er die seit der Gründung dieser Kommission im Jahr 2011 durchgeführten und für die Jahre 2014 und 2015 geplanten Projekte vorstellte.

Als für Österreich besonders erfreulich kann vermerkt werden, dass es bei dieser Sitzung mit kräftiger Unterstützung der Kollegen der Deutsch- und Niederländisch-sprechenden Abteilung (Dutch- and German-speaking Division, DGSD) - allen voran Pier-Giorgio ZACChEDDU (Deutschland) und Ferjan ORMELING (Niederlande) - und der früheren UNGEGN-Vorsitzenden Helen Kerfoot (Kanada) mit ihrer immer noch gewichtigen Stimme gelungen ist, mit dem Übergang von Hubert Bergmann auf Gerhard Rampl die internationale Koordination der Toponymic Guidelines in österreichischer Hand zu behalten. Von den zwölf Funktionen der operativen Führung der UNGEGN besetzt Österreich somit weiterhin zwei.

Besonderer Dank ist auch der Ständigen Vertretung Österreichs bei den Vereinten Nationen in New York, speziell Herrn Botschafter Dr. Martin S AJDIK und Herrn Botschaftsrat Dr. Hannes MACHOR auszusprechen, mit denen enger Kontakt gehalten wurde und die vorzügliche logistische und mentale Unterstützung leisteten.

\section{Tagung der UNGEGN-Arbeitsgruppe für Exonyme (UNGEGN Working Group on Exonyms, WGE), Hermagor, 5.-7. Juni 2014}

Die UNGEGN-Arbeitsgruppe für Exonyme hat seit ihrer Gründung im Jahr 2002 eine Tradition eigener Tagungen mit Workshop-Charakter zusätzlich zu den Business Meetings bei UNGEGN-Sitzungen und Konferenzen zur Standardisierung geographischer Namen entwickelt. So wurden derartige Workshops bisher im Jahr 2003 in Prag [Praha], 2005 in Laibach [Ljubljana], 2007 wieder in Prag, 2008 in Temeswar [Timişoara], 2010 in Tainach/Kärnten, 2012 in Danzig [Gdańsk] und 2013 in Korfu veranstaltet. Stets stand ein Schwerpunktthema zur Diskussion wie die Frage der Kriterien für den Gebrauch von Exonymen oder die begriffliche Abgrenzung von Endonym und Exonym. 
Das 16. Arbeitstreffen fand nun in Hermagor (Kärnten), im Heimatort des Convenors und von ihm organisiert, statt. Hauptthema war weiterhin die Diskussion allgemeingültiger Definitionen des Endonyms und des Exonyms, die bei diesem Treffen zu einem Abschluss gebracht werden sollte. An der Tagung nahmen 28 Experten aus 17 Ländern teil (vgl. auch Abb. 1), die insgesamt 18 Vorträge hielten. Aus Österreich beteiligten sich außer dem Convenor der Arbeitsgruppe, Peter JoRDAn, noch der Historiker Michael DuschaneK (Niederösterreichische Landesbibliothek Sankt Pölten), die Linguisten Isolde HAuSNER (ÖAW Wien) und Heinz-Dieter Pohl (Universität Klagenfurt a.W.) sowie der freiberuflich tätige Kartograph und Toponomast Roman STANI-FERTL (Kritzendorf). Eine ausführliche Diskussion schloss tatsächlich die nun schon jahrelange Debatte um die Definitionen des Begriffspaars Endonym/Exonym ab - allerdings mit dem Ergebnis, dass die bereits seit 2007 geltenden Definitionen des UNGEGN-Glossars als das realpolitisch erreichbare Optimum erachtet wurden. Die jahrelange und tiefschürfende Debatte um die beiden Begriffe hat diese aber unter allen möglichen Aspekten ausgeleuchtet und ist in vier Tagungsbänden ${ }^{2}$ dokumentiert, die als ein bleibendes Ergebnis der Arbeitsgruppe

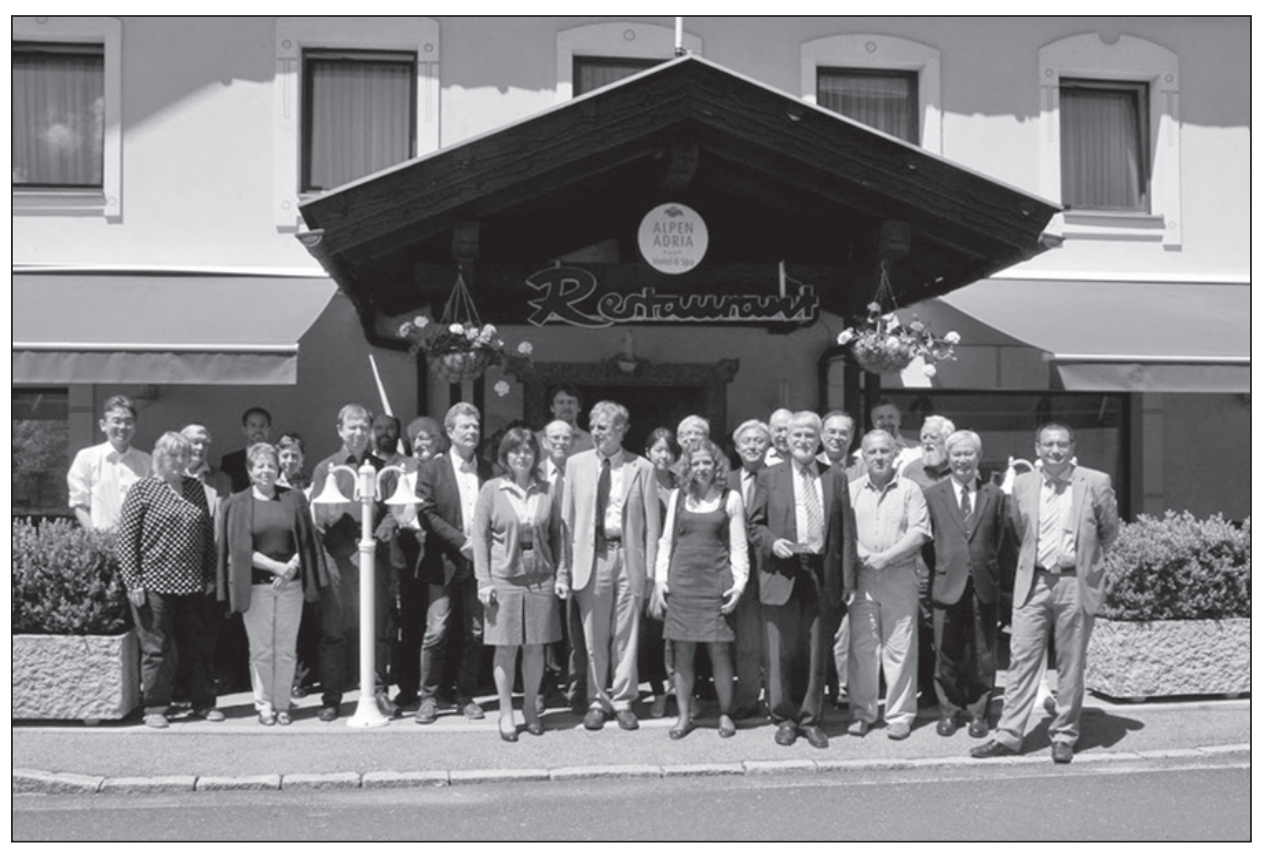

Abb. 1: Die Teilnehmer der 16. Tagung der UNGEGN-Arbeitsgruppe für Exonyme vor dem Konferenzhotel am Presseggersee bei Hermagor (Foto: Maciej ZYCH)

2) Jordan P., Orožen Adamič M., Woodman P. (Hrsg.) (2007), Exonyms and the International Standardisation of Geographical Names. Approaches towards the Resolution of an Apparent Contradiction (= Wiener Osteuropa-Studien, 24). Münster, LIT.

Jordan P., Bergmann H., Burgess C., Cheetham C. (Hrsg.) (2011), Trends in Exonym Use. Proceedings of the $10^{\text {th }}$ UNGEGN Working Group on Exonyms Meeting, Tainach, 28-30 April 2010 (= Name \& Place, 1). Hamburg, Dr. Kovač.

Woodman P. (Hrsg.) (2012), The Great Toponymic Divide. Reflections on the definition and usage of endonyms and exonyms. Warszawa, Head Office of Geodesy and Cartography.

Jordan P., Woodman P. (Hrsg.) (2013), The Quest for Definitions. Proceedings of the $14^{\text {th }}$ UNGEGN Working Group on Exonyms Meeting, Corfu, 23-25 May 2013 (= Name \& Place, 3). Hamburg, Dr. Kovač. 
angesehen werden können. Eine weitere Dokumentation dieser Diskussion wird in Form der Abhandlungen der Tagung in Hermagor als Band 4 der toponomastischen Buchreihe Name \& Place erscheinen, die von Peter JoRdan und Paul Woodman herausgegeben wird.

Die Tagung klang bei prachtvollem Wetter mit einer toponymisch akzentuierten Wanderung entlang des nahe gelegenen Weißensees aus. Zur Begrüßung in Hermagor hatten sich außer dem Bürgermeister der Stadtgemeinde Hermagor-Presseggersee, Siegfried RonACHER, auch die Kärntner Landeshauptmann-Stellvertreterin Gabriele ScHAUnIG eingefunden.

Näheres zu Inhalt und Teilnahme an der Tagung ist auf der Website der Arbeitsgruppe zu finden: http://ungegn.zrc-sazu.si/

\section{Internationaler Kongress zur Namenforschung, Glasgow, 25.-29. August 2014}

Die Kongresse dieser Serie finden alle drei Jahre statt und sind die Weltkongresse der Onomastik, der Wissenschaft(en) von den Eigennamen, also nicht nur von den geographischen Namen, sondern auch von Personennamen, Namen von Tieren, Pflanzen usw. Sie stehen unter dem Schirm des International Council of Onomastic Sciences (ICOS), der im Gegensatz zur UNGEGN, in der Geographen und Kartographen in der Mehrheit sind und auch den Ton angeben, von der Linguistik dominiert wird. Als toponomastisch tätiger Geograph nimmt man an diesen Kongressen unter anderem teil, um sich mit seinen (kultur-)geographischen Thesen einer linguistischen Kritik zu stellen und zu testen, ob diese Thesen auch vor Linguisten bestehen können. Tatsächlich ist das linguistische Interesse an geographischen Sichtweisen sehr groß, und auch unter Linguisten wächst die Beschäftigung mit Raumbezügen. So gab es beim letzten ICOS-Kongress in Barcelona im Jahr 2011 erstmals eine (vom Verfasser geleitete) Sektion „Onomastik und Geographie“, die zahlreich mit Vorträgen besetzt und sehr gut besucht war. Zwar wurde dieser Ansatz in Glasgow nicht fortgeführt, weil das Programm prinzipiell nicht in Sektionen gegliedert war, doch fand sich ein mächtiger Strang toponomastischer Sitzungen, der sich oft sogar in Parallelveranstaltungen aufspaltete. Das Spektrum der Themen reichte von einem Vergleich der Benennungsmotive für geographische Objekte bei verschiedenen indigenen Gruppen bis zur Bedeutung von Straßennamen für die raumbezogene Identität von Großstadtbewohnern.

Der Kongress in Glasgow hatte etwa 300 Teilnehmer, was allerdings nur etwa einem Drittel der Teilnehmerzahl des 24. Kongresses in Barcelona entsprach. Aus Österreich waren fünf Onomasten gekommen, zwei davon hielten Vorträge. Der Linguist Hubert Bergmann sprach über „House-names litanies - a phenomenon at the intersection between onomastics and ethnography“, Peter JoRDAn stellte das sehr prinzipielle Thema „Place names as an expression of human relations to space“" zur Diskussion.

\section{Symposion über Änderungen geographischer Namen, Rom [Roma], 17.-18. November 2014}

Sprachen werden von politischen Mächten gefördert und sind von ihnen abhängig. In historischer Dimension gibt es ein Kommen und Gehen von Sprachen - je nachdem, ob sie politisch unterstützt werden oder ihnen diese Unterstützung wieder verloren geht. Geographische Namen als Elemente der Sprache sind Teile dieses historischen Wandels. Wegen ihrer symbolischen Wirkung als Markierungen geographischer Objekte, von Territorien und raumbezogenen Identitäten sind sie jedoch noch stärker dem Wandel unterworfen. Bereits der Wechsel des politischen Mainstreams in einer Gesellschaft kann zum Austausch geographischer Namen führen. Ein aktuelles Beispiel sind Aufstieg und Fall des Kommunismus im östlichen Europa. Aber auch die 
Kommerzialisierung unserer Gesellschaften hat ihre Auswirkungen auf die Namenlandschaft. Kommerzielle Namen ersetzen manchmal traditionelle, orts- und umgebungsbezogene, was die Frage aufwirft, ob geographische Namen nicht als ein wichtiger Teil des kulturellen Erbes angesehen und aus diesem Grund geschützt werden sollen.

Fast vollständige Inversionen der Namenlandschaft - wie nach dem Ende der Apartheid in Südafrika oder den Anschluss Südtirols an Italien nach dem Ersten Weltkrieg - sind sicherlich durch den Wechsel der politischen Dominanz verursacht. Es können aber auch neue kulturelle Strömungen und Haltungen innerhalb einer sich ansonsten nicht markant verändernden Gesellschaft zu Änderungen in der Namenlandschaft führen - wie zum Beispiel schon seit einiger Zeit in Kanada, wo den Sprachen und Namen indigener und nicht-dominanter Gruppen vermehrt Aufmerksamkeit geschenkt wird. Die geographischen Namen der neu kodifizierten indigenen Sprachen werden standardisiert und für amtlich erklärt und somit im öffentlichen Raum erstmals sichtbar.

Änderungen geographischer Namen betreffen sehr oft Straßennamen - oder allgemeiner formuliert: Namen im städtischen Raum, da viele von ihnen Gedenkfunktion haben und so eng mit historischen Epochen und Mächten in Zusammenhang stehen. Aber auch Namen von Siedlungen und besonders Städten unterliegen oft Veränderungen - wegen der Prominenz dieser Objekte und ihrer Bedeutung in Politik, Kultur und Wirtschaft. Ganz besondere Fälle sind Namen von Verwaltungseinheiten aller räumlichen Ebenen. Sie sind besonders eng mit dem jeweils herrschenden politischen System verbunden und entsprechend Änderungen ausgesetzt.

Das Thema dieses Symposions war also sehr raumbezogen und geographisch. Es hatte aber auch wesentliche historische, soziologische und politische Aspekte, während linguistische Sichtweisen eher in den Hintergrund traten.

Als Veranstalter fungierten die Joint IGU/ICA Commission/Working Group on Toponymy sowie die Accademia Nazionale dei Lincei, die italienische Akademie der Wissenschaften, in deren Sitz, der prachtvollen Villa Farnesina am rechten Ufer des Tiber [Tevere], das Symposion auch stattfand. Peter JoRDAN organisierte die Tagung im Auftrag der Joint IGU/ICA Commission/ Working Group inhaltlich und in Bezug auf den Teilnehmerkreis, Cosimo Palagiano (Rom) war für die örtliche Organisation verantwortlich. Die 50 Teilnehmer kamen aus 21 Ländern und von vier Kontinenten. Es wurden 40 Vorträge gehalten, darunter zwei von Österreichern: Roman Stani-Fertl (Kritzendorf) sprach über „Place-names changes and the European Location Framework (ELF)“, Peter Jordan (Wien) zum Thema „The ambivalent view on Tito in the successor states of Yugoslavia reflected by the preservation or replacement of place names commemorating him".

Die Abhandlungen des Symposions werden als Band 5 der toponomastischen Buchreihe Name \& Place erscheinen. 